\title{
Polarimetric and Spectroscopic Observations of a Dynamically New Comet C/2012 J1 (Catalina)
}

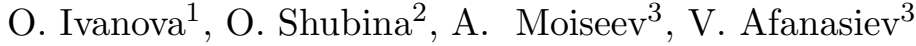 \\ ${ }^{1}$ Main Astronomical Observatory, National Academy of Sciences, Kyiv, 03680 Ukraine \\ ${ }^{2}$ Taras Shevchenko National University of Kyiv, Kyiv, 01601 Ukraine \\ ${ }^{3}$ Special Astrophysical Observatory, Russian Academy of Sciences, Nizhnij Arkhyz, 369167, Russia
}

January 5, 2015/May 27, 2015

\begin{abstract}
We present the analysis of the polarimetric and spectral data obtained for the dynamically new comet C/2012 J1 (Catalina) when it was at a distance of 3.17 AU from the Sun. The observations were made at the prime focus of the 6-m BTA telescope using the SCORPIO-2 focal reducer. The map of the distribution of linear polarization in the cometary coma was constructed. The calculated value of linear polarization was on average about $-2 \%$. Spectral analysis of the cometary coma allowed us to detect the emission of the CN molecule in the $(0-0)$ band. The gas production rate was derived using the Haser model and amounted to $3.7 \times 10^{23}$ molecules per second.
\end{abstract}

\section{INTRODUCTION}

The comets, minor planets, meteoroid particles, and interplanetary dust form an evolutionarily interconnected family of small bodies of the Solar System. Despite their modest masses, research of these objects is very important from the cosmogonic point of view, namely, to uncover the origin of the Solar System. According to modern cosmological ideas, comets are the relics of the Solar System, and their composition should be most similar to that of the primordial protoplanetary cloud. The question about parameters of dust particles (their size, orientation, structure) in comas and tails of comets active at large heliocentric distances and the dynamically new comets, which for the first time enter the inner regions of the Solar System, remains unsolved. At heliocentric distances greater than $3 \mathrm{AU}$, the matter is carried out from the surface of the cometary nucleus due to sublimation of water ice and more volatile components $\left(\mathrm{CO}, \mathrm{CO}_{2}\right)$, the characteristic and outflow velocity of matter are also varying. The question of variation of dust scattering properties with heliocentric distance of comets also remains open. Available observational data indicate a difference between activity of dynamically new comets (active at large heliocentric distances) and shortperiod comets (Epifani et al., 2007, 2008; Korsun et al., 2008, 2010, 2014). Hence, it is possible that the nature of dust particles differs as well.

Observations of comets using different methods (photometry, spectroscopy, and polarimetry) and at different

Send offprint requests to: Oleksandra Ivanova e-mail: sandra@mao.kiev.ua heliocentric distances and phase angles provide useful information on the material of the comet surface layer: the particle sizes, their structure, and chemical composition. In this paper, we study a dynamically new comet C/2012 J1 (Catalina) using polarimetric and spectroscopic methods.

Comet C/2012 J1 (Catalina) was discovered on May 13, 2012 as a 16.4 object within the Catalina Sky Survey (Apitzsch et al., 2012), a project searching for new comets and asteroids. The comet revealed significant activity at a distance larger than $3 \mathrm{AU}$. It passed the perihelion on December 7, 2012 at a distance of 3.15 AU. The comet belongs to the class of long-period comets and has a parabolic orbit with eccentricity $e=1.001$ and orbit inclination $i=34$. 1 .

\section{OBSERVATIONS}

Our polarization and spectroscopic observations were carried out on November 15, 2012, when the comet was at a distance of 3.17 AU from the Sun and 2.45 AU from Earth, and its integral magnitude was 13.9 . The phase angle of the comet was $14^{\circ}$, its scale in the sky plane was approximately $1800 \mathrm{~km} /$ arcsec. The observations were performed on the 6-m BTA telescope of the Special Astrophysical Observatory of the Russian Academy of Sciences (SAO RAS) using the multimode SCORPIO-2 focal reducer (Moiseev, 2011) operating in the spectroscopic and polarimetric modes. An E2V 42-90 CCD sized $4600 \times 2048$ pixels was used as a detector. The size of the 
Table 1.

Log of Comet C/2012 J1 (Catalina) observations

\begin{tabular}{c|c|c|c|c|c|c|c}
\hline $\begin{array}{c}\text { Start time, } \\
\text { UT }\end{array}$ & $\begin{array}{c}r, \\
\mathrm{au}\end{array}$ & $\begin{array}{c}\Delta, \\
\mathrm{au}\end{array}$ & $\begin{array}{c}\alpha, \\
\mathrm{deg}\end{array}$ & $\begin{array}{c}\text { PA, } \\
\mathrm{deg}\end{array}$ & Grating/filter & $\begin{array}{c}\text { Total exposure, } \\
\mathrm{s}\end{array}$ & Data \\
\hline Nov 15.8026, 2012 & 3.17 & 2.45 & 14.02 & 87.66 & $V$ & 15 & image \\
Nov 15.8092, 2012 & 3.17 & 2.45 & 14.02 & 87.66 & VPHG1200@540 & 1500 & spectrum \\
Nov 15.8280, 2012 & 3.17 & 2.45 & 14.02 & 87.66 & $V$ & 640 & image/polarization \\
\hline
\end{tabular}

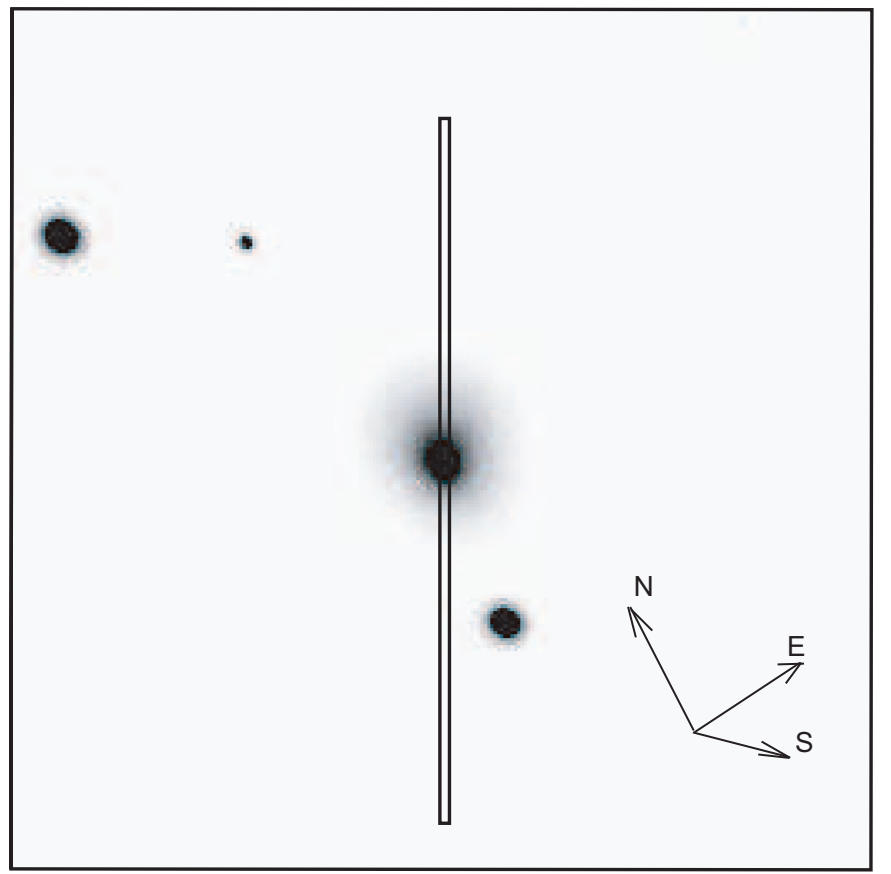

Fig. 1. An image of Comet C/2012 J1 (Catalina) in the $V$ filter and the projection of the spectrograph slit on the cometary coma at the time of observation. The directions to north, east, and to the Sun are marked.

field of view was $6.1 \times 6{ }^{\prime} 1$, the scale in the $2 \times 2$ hardware binning mode amounted to 0 "' 18 per pixel.

Polarimetric observations were carried out in the broadband $V$ filter of the Johnson-Cousins photometric system. A polarizing dichroic filter (Polaroid) was used as a polarization analyzer.

Spectroscopic observations were carried out in the long slit mode. The height of the slit amounted to $6 ! 1$, the slit width was $1^{\prime \prime}$. The grism with a volume-phase holographic grating VPHG1200@540 provided the wavelength range of 3600-7200 $\AA$ with a spectral resolution of about $5.2 \AA$. The observing $\log$ is given in the table. Figure 1 shows a $V$-band image of the comet and the projection of the spectrograph slit onto the cometary coma.

\section{POLARIMETRIC INVESTIGATION}

To estimate the degree of linear polarization of the comet, we used the dichroic polarization analyzer installed in the spectrograph. The analyzer was positioned at the three fixed angles: $0^{\circ}$ and $\pm 60^{\circ}$. We obtained the intensity values for these angles $I(x, y)_{0^{\circ}}, I(x, y)_{-60^{\circ}}$, and $I(x, y)_{+60^{\circ}}$ and estimated the Stokes $Q^{\prime}$ and $U^{\prime}$ parameters at each point of the image:

$$
\left\{\begin{array}{l}
Q^{\prime}=\frac{2 I(x, y)_{0} \circ-I(x, y)_{-60^{\circ}} \circ-I(x, y)_{+60^{\circ}}}{I(x, y)_{0} \circ+I(x, y)_{-60}{ }^{\circ}+I(x, y)_{+60^{\circ}}}, \\
U^{\prime}=\frac{\sqrt{3}}{2} \frac{I(x, y){ }_{+60} \circ-I(x, y)_{-60} \circ}{I(x, y)_{0} \circ+I(x, y)_{-60^{\circ}} \circ+I(x, y)_{+60^{\circ}}} .
\end{array}\right.
$$

The true values of the Stokes $Q$ and $U$ parameters were obtained from the following equations:

$$
\begin{aligned}
& U=U^{\prime} \cos 2 \varphi-Q^{\prime} \sin 2 \varphi, \\
& Q=U^{\prime} \sin 2 \varphi+Q^{\prime} \cos 2 \varphi .
\end{aligned}
$$

To determine the degree of polarization $P$ and the angle of the polarization plane $\varphi$, we used the following relations:

$$
\left\{\begin{array}{l}
P=\sqrt{Q^{2}+U^{2}}, \\
\mathrm{PA}=\frac{1}{2} \arctan \frac{U}{Q} .
\end{array}\right.
$$

Reduction of polarimetric images included subtraction of the bias frame, flat field correction, and formation of images prepared for processing. Next, using the central isophotes of the comet image, all the images were reduced to a single photometric center. To improve the signal-to-noise ratio, the corrected frames with the comet image were added up using the median averaging procedure. A detailed description of the technique of observation and data reduction with the SCORPIO2 spectrograph in the polarimetric mode is described in Afanasiev \& Amirkhanyan (2012).

Using formulas (11)-(3), we obtained the degree and angle of linear polarization for Comet C/2012 J1 (Catalina). Figure 2 presents the distribution of linear polarization over the coma, projected onto the scattering plane.

We have also made sections of the linear polarization of the comet in the direction to the Sun and in the direction perpendicular to it (Fig. 3). The sections were made through the photometric center of the image.

According to our estimates, the linear polarization over the cometary coma (inside the $\rho<17^{\prime \prime}$ radius, which corresponds to approximately $30000 \mathrm{~km}$ ) is on average $-2.03 \% \pm 0.1 \%$. The map of spatial distribution and the demonstrated cross-sections (Figs. 2 and 3) show that the nuclear region (up to $15000 \mathrm{~km}$ ) reveals no abrupt changes in the value of linear polarization. We do not consider the area smaller than $5000 \mathrm{~km}$, which is poorly resolved. In addition, there may also occur errors due to the overlap 


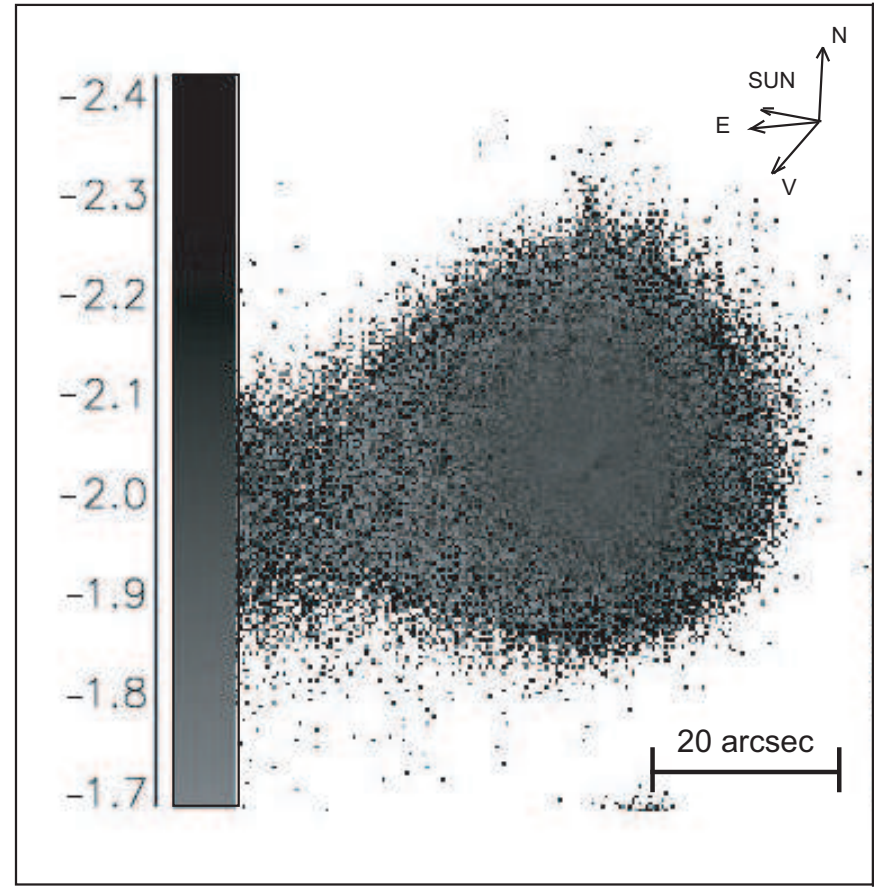

Fig. 2. Distribution of linear polarization over the coma, projected onto the scattering plane. The scale of the image is marked.

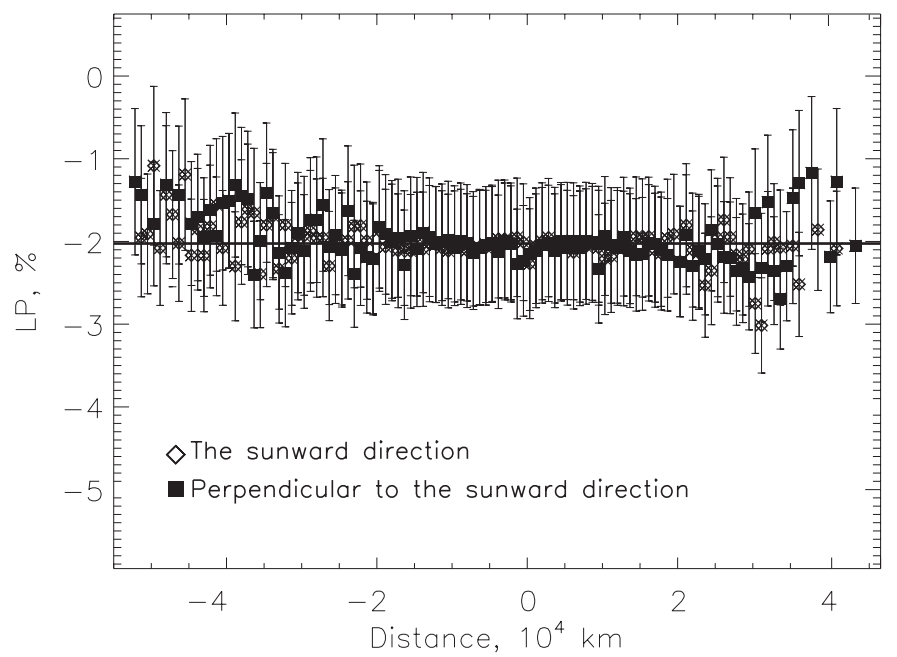

Fig. 3. Cross sections of the linear polarization of the comet (LP).

of different images. In the field with a radius of more than $25000 \mathrm{~km}$, we possibly observe (in the absolute values) a small decrease of the linear polarization.

\section{EXAMINATION OF THE COMETARY SPECTRUM}

Primary reduction of spectral data was performed using the codes developed at SAO RAS, operating in the IDL environment. It included the bias frame subtraction, removal of cosmic ray traces, flat field correction with the spectrum of a built-in lamp with a continuous spectrum,

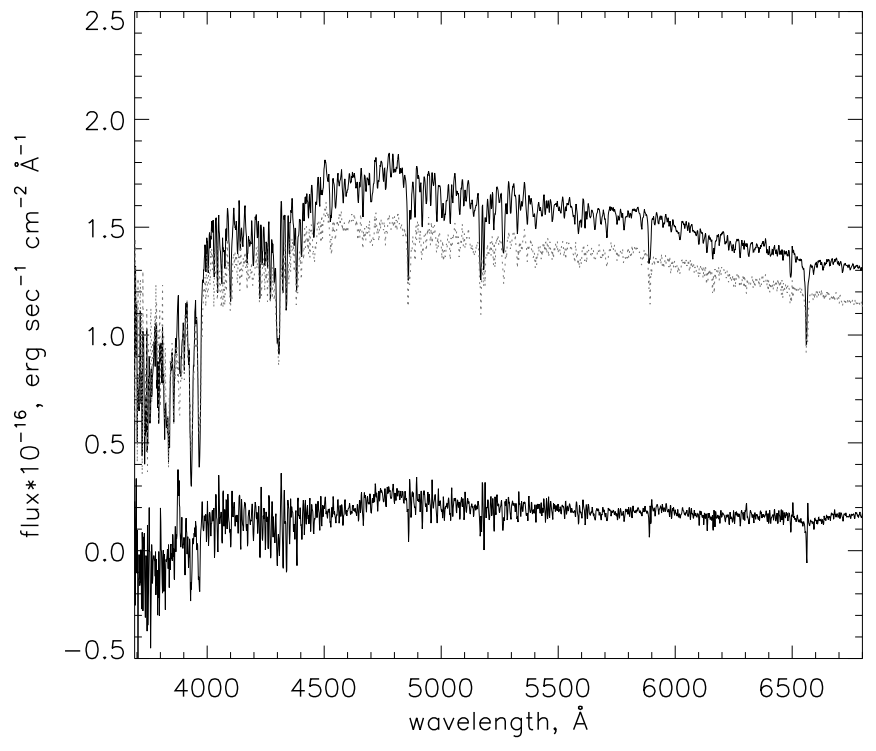

Fig. 4. The spectrum of the comet (the black curve), the modified solar spectrum (the bright dashed line), and the result of its subtraction from the initial spectrum.

spectral line curvature compensation, wavelength calibration, and representation of the data in an evenly spaced wavelength scale. The wavelength calibration of the spectra was carried out using a He-Ne-Ar lamp.

To convert the cometary spectra into absolute power values, we used the spectra of the standard star $\mathrm{BD}+28^{\circ} 4211$ (Oke, 1990), which was observed at the same night. The response curves of the Earth's atmosphere for SAO RAS from the paper of Kartasheva and Chunakova (Kartasheva \& Chunakova, 1978) were applied.

To analyze the energy distribution in the cometary spectrum and search for possible molecular emissions, a one-dimensional spectrum was built by adding up the counts along the slit within $\pm 18^{\prime \prime}$ from the nucleus.

To isolate the emission spectrum of the comet, we subtracted from the cometary spectrum the high-resolution solar spectrum (Neckel \& Labs, 1984), which had previously been convoluted with a Gaussian function with an FWHM corresponding to the width of the instrumental profile in our observations. The solar spectrum was scaled the way that its level was as close as possible to the low limit of the cometary spectrum in the spectral intervals dominated by the continuum. In addition, we introduced a correction for the reddening effect. The scale of the observed wavelengths was corrected for the Doppler shift of the cometary spectrum (radial velocity of about $22 \mathrm{~km} / \mathrm{s}$ ). The qualitative analysis of the cometary spectrum revealed the presence of weak molecular emissions. The total spectrum of Comet C/2012 J1 (Catalina) is presented in Fig. 4.

Identification of molecular emissions, observed in comets in this spectral range, was carried out by comparing the spectrum of the comet with the calculated 


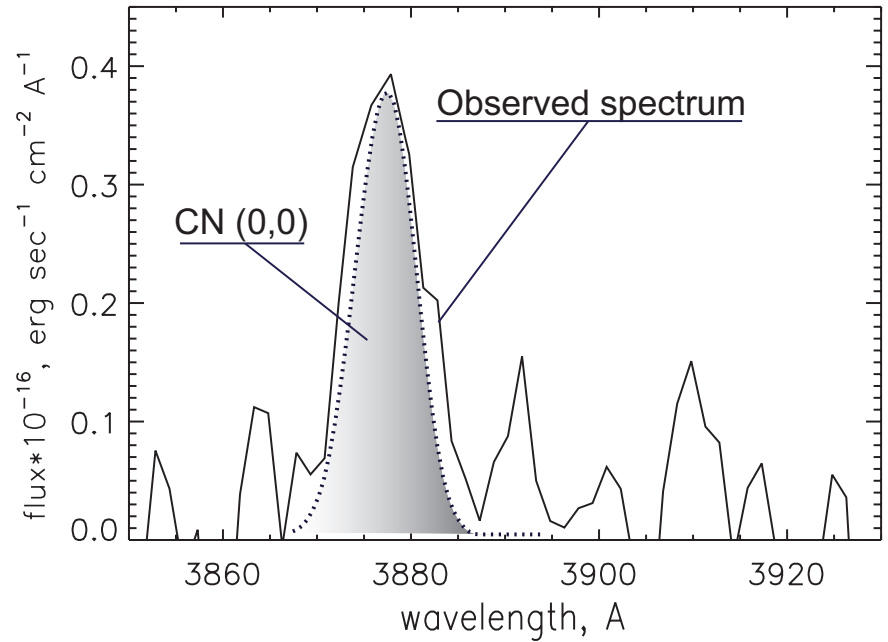

Fig. 5. Calculated and identified profile of the $\mathrm{CN}$ molecule (0-0) band.

and laboratory spectra of molecules. The spectrum reveals the presence of molecular emissions as well as differences in the energy distribution of the cometary and the solar spectra. Although the blue spectral region is very noisy, we managed to identify several spectral features which belong to the $\mathrm{CN}$ molecule (electronic transition $\left.\mathrm{B}^{2} \Sigma^{+}-\mathrm{X}^{2} \Sigma^{+}\right)$. Since the rotational structure of the band in the resulting spectrum has not been resolved, we could only identify the edges of the oscillating system (00 ) bands. The theoretical spectrum for this molecule was built using the LIFBASE code (Luque \& Croslev, 1999), which is used to calculate electronic spectra of diatomic molecules. Figure 5 shows the identified spectral detail.

Assuming that the atmosphere of the comet is optically thin (in this case, the fluxes are directly proportional to the number of radiators), we can estimate the total number $N(\rho)$ of molecules contained in a column of radius $\rho$ on the line of sight using the formula:

$N(\rho)=\frac{L}{g_{\lambda}}$,

where $L=4 \pi \Delta^{2} F_{c}$ is the observed luminosity of the comet in the emission band, $F_{c}$ is the flux of the comet in this band, $\Delta$ is the geocentric distance, $g_{\lambda}$ is the efficiency of fluorescence for the given molecule at the heliocentric distance $r=1 \mathrm{AU}$, which is determined by the expression (Osterbrock, 1962):

$g_{\lambda}=\frac{\pi e^{2}}{m_{e} c^{2}} \lambda^{2}\left(\pi F_{\operatorname{Sun} \lambda}\right) f_{\lambda} w$

where $m_{e}$ and $e$ are the mass and the charge of the electron, $c$ is the speed of light, $f_{\lambda}$ is the oscillator strength, $w$ is the probability of vibrational transition, $\pi F_{\operatorname{Sun} \lambda}$ is the density of solar radiation at heliocentric distance $r$.
To convert the observed density of molecules in the column $N(\rho)$ into the molecule production $Q$, we used the Haser model (Haser, 1957), according to which

$$
\begin{aligned}
& N(\rho)=\frac{Q}{v_{g}} \rho\left[\int_{x}^{x \mu} K_{0}(y) \mathrm{d} y\right. \\
& \left.+\frac{1}{x}\left(1-\frac{1}{\mu}\right)+K_{1}(x \mu)-K_{1}(x)\right],
\end{aligned}
$$

where $v_{g}$ is the speed of molecule fly-out, $\mu=\gamma_{p} / \gamma_{d}$, $x=\gamma_{d} d, \gamma_{p}$ and $\gamma_{d}$ are the characteristic path lengths of the parent and the daughter molecules respectively, $K_{0}$ and $K_{1}$ are the modified zero- and first-order Bessel functions of the second kind.

Model calculations involve the full flux inside a specified aperture. Since in our observations we only register the spectrum from the regions captured by the slit of the spectrograph, a corresponding correction needs to be done. When calculating the aperture correction, we took into account both the ratio between the slit areas and the circular aperture and the observed brightness distribution along the slit.

For our calculations, we used the following values of model parameters. The characteristic scales for the parent and daughter molecules are $3.0 r^{1.3} \times 10^{4} \mathrm{~km}$ and $19.9 r^{0.6} \times 10^{4} \mathrm{~km}$ respectively (Langland-Shula \& Smith, 2011). Since the $g$-factor for the $\mathrm{CN}(0-0)$ band is dependent on the heliocentric velocity of the comet, its value at the time of observation was refined using the calculations made in Schleicher (2010). In our case it was $2.4 \times 10^{-13} r^{-2} \mathrm{erg} \mathrm{s}^{-1}$ per molecule. The rate of gas outflow was assumed to be the value which is used in most of such calculations, $1 \mathrm{~km} \mathrm{~s}^{-1}$ (Langland-Shula \& Smith, 2011; Schulz \& IAUSymp, 2005).

For the accepted values the $\mathrm{CN}$ gas production amounted to $3.7 \times 10^{23}$ molecules $/ \mathrm{s}$.

\section{DISCUSSION}

Research of the properties of gas and dust in comets with different perihelion distances allows us not only to estimate the possible differences or similarity of the cometary material detected at different heliocentric distances but also to draw some conclusions about physical conditions of comet formation in general. Complex research of various comets, namely, regular monitoring of their spectra for the purpose of detection of gas emissions, registration of the onset of formation of these emissions as a function of heliocentric distance as well as investigation of polarization distribution of gas and dust in the comets, gives the possibility of building a realistic model of the cometary nucleus. The short-period comets and a number of long-period comets with perihelia shorter than $2 \mathrm{AU}$, for which enough observed spectral, photometric, and polarimetric material 
was accumulated, are by now the most thoroughly studied. New observational strategies and the use of large telescopes has recently allowed the active research of objects from distant outskirts of the Solar System. Focusing on the research of properties of dynamically new comets that arrive in the inner Solar System for the first time, we would make a comparative analysis aimed to classify the groups of comets by physical properties of dust and gas, which are directly linked to the evolution and/or places of origin.

The aim of our polarimetric and spectroscopic observations was to study a dynamically new comet C/2012 J1 (Catalina). During the analysis of polarimetric data, we measured the magnitude of linear polarization. Its average value over the cometary coma varies from $-1.9 \%$ to $-2.1 \%$. Polarization of scattered radiation depends on the heliocentric distance, phase angle, and morphology of the coma, which is directly connected with the activity of the comet. Many comets, including the distant ones, reveal jet activity, the consequence of which may also be the variation of polarization properties of the dust particles of the coma. Due to the jets, the nuclear region may reveal areas with different polarization.

Polarization is also dependent on the diameter of the aperture within which the measurements were made. The aperture dependence appears in general case due to different contributions of the continuum and the gas emissions into the integral radiation. For this reason, we can only compare polarization in different comets (assuming equal remaining parameters: similar fields, cut by the filters, comets without emissions) in cases of similar sizes of the measured coma. In our study, we did not use the apertures for observations, and the broadband filter that we applied did not capture any bright molecular emissions.

The constructed polarization map allowed us to explore different regions of the cometary coma. The analysis of the distribution map, complemented by the plotting of polarization sections performed in the direction to the Sun and in the direction perpendicular thereto, indicated that linear polarization does not essentially vary over the cometary coma. Our observations do not reveal any regions with different polarization. Based on this, it can be assumed that the coma of Comet C/2012 J1 (Catalina) has no isolated jet structures as in Comet 29P/SchwassmannWachmann 1 (Ivanova et al., . 2014), or a fan-shaped tail as in Comet 2P/Encke (Kiselev et al., 2004), which can lead to the occurrence of regions with different polarization.

If we compare the values of linear polarization given in the literature for the dust comets, our value proves to be the highest by the absolute value. For example, the degree of polarization was $-1.49 \%$ (at a phase angle of $\left.11^{\circ}\right)$ for Comet 1P/Halley, $-1.09 \%(\varphi=14.8)$ for Comet C/1989X1 (Austin), $-1.4 \%$ ( $\varphi=12.56)$ for $47 \mathrm{P} /$ Ashbrook-Jackson, $-1.78 \%(\varphi=15.7)$ for C/1990 K1 (Levy) Rosenbush, 2006). But this difference in the degree of polarization can be related to the fact that our value was calculated for the entire coma, while those given for comparison are for the apertures. However, in some distant comets with perihelia of more than $4 \mathrm{AU}$ which are active over long distances and virtually without emissions (Korsun et al., 2008, 2010), the linear polarization was also higher than for the comets that were observed at close distances from the Sun at the same phase angles (Ivanova et al., . 2014). In this case, polarization variations can be caused by the conditions that lead to activity of the comet at different distances from the Sun, as well as by the rate of dust loss, which is also different for distant comets and comets observed at a distance of less than $2 \mathrm{AU}$.

The spectroscopic observations of Comet C/2012 J1 (Catalina) registered the CN molecule emission in the (0-0) band of its violet system. At a distance of more than $3 \mathrm{AU}$, most of the cometary spectra are poor in emissions. But in a number of distant comets, emission features of the $\mathrm{CN}$ molecule were observed at large heliocentric distances (Korsun et al., 2006, 2008, 2014). The cometary spectra (e.g., for Comet C/1995 O1 (Hale-Bopp)) at such distances also reveal the $\mathrm{C}_{2}$ molecule emissions (A'Hearn et al., 1995; Hicks, 1996), but they were not identified in the spectrum of our comet.

Measuring the flux in the $\mathrm{CN}$ emission, we estimated the rate at which these molecules arrive into the cometary coma. According to our calculations, gas production amounts to $3.7 \times 10^{23}$ molecules/s for the CN molecule. Comparing our results with the data from Langland-Shula \& Smith (2011), we can conclude that the $\mathrm{CN}$ molecule gas production is close to the lower limit of this value for the majority of comets observed at heliocentric distances closer than 2 AU., indicating low gas production of the comet.

\section{CONCLUSION}

The analysis of polarimetric and spectral observations of the dynamically new comet C/2012 J1 (Catalina), performed on the SAO RAS 6-m telescope, suggests the following conclusions:

(1) the average value of linear polarization (projected onto the scattering plane) ranges from $-1.9 \%$ to $-2.1 \%$;

(2) the spectrum of the comet revealed the $\mathrm{CN}$ molecule emission in the (0-0) band of its violet system;

(3) gas production for the $\mathrm{CN}$ molecule is $3.7 \times 10^{23}$ molecules $/ \mathrm{s}$;

Acknowledgements. The observations on the 6-m BTA telescope were conducted with financial support of the Ministry of Education and Science of the Russian Federation (agreement no. 14.619.21.0004, project ID RFMEFI61914X0004). The authors thank P. P. Korsun for critical comments and discussion, as well as the anonymous referee for comments that helped to improve the article. A. Moiseev thanks the non-profit Dynasty foundation for financial support. 


\section{References}

A'Hearn M.F., Millis R.L., Schleicher D.G., et al., 1995, Icarus 118, 223

Afanasiev V.L., Amirkhanyan V.R., 2012, Astrophysical Bulletin 67, 438

Apitzsch R., Bill H., Gibbs A.R., et al., 2012, Minor Planet Electronic Circ., 2012-J49

Luque J., Crosley D. R. , 1999, SRI International Rep., MP 99-009

Epifani E.M., Palumbo P., Capria M.T., et al., 2007, MNRAS381, 713

Epifani E.M., Palumbo P., Capria M.T., et al., 2008,MNRAS390, 265

Haser L., 1957, Bull. Class Sci. Academie Royale de Belgique 43, 740

Hicks U.FinkandM.D., 1996, ApJ459, 729

Ivanova A., et al., 2014, in Proc Int. Conf. on Asteroids, Comets, Meteors (ACM 2014), Helsinki, Finland, 2014 (Univ. of Helsinki, Helsinki, 2014) p. 251.

Kartasheva T.A., Chunakova N.M., 1978, Astrofizicheskie Issledovaniia 10, 44

Kiselev N.N., Jokers K., Bonev T., 2004, Icarus 168, 385

Korsun P.P., Ivanova O.V., Afanasiev V.L., 2006, A\&A459, 977

Korsun P.P., Ivanova O.V., Afanasiev V.L., 2008, Icarus 198, 465

Korsun P.P., Kulyk I.V., Ivanova O.V., et al., 2010, Icarus 210, 916

Korsun P.P., Rousselot P., Kulyk I.V., et al., 2014, Icarus 232, 88

Neckel H., Labs D., 1984, Solar Physics 90, 205

Afanasiev V.L., Moiseev A.V., 2011, Baltic Astronomy 20, 363

Oke J.B., 1990, AJ99, 1621

Osterbrock C.R.O'dellandD.E., 1962, ApJ136, 559

Rosenbush V. K., 2006, Doctoral Dissertation in Mathematics and Physics (Main Astron. Obs., Kyiv)

Schleicher D.G., 2010, AJ140, 973

Schulz R., et al., 2005, No. 229, 413

Langland-Shula L.E., Smith G.H., 2011, Icarus 213, 280 